\title{
A presença de alcalóides em espécies botânicas da Amazônia. III - Annonaceae (1)
}

\author{
A. I. da Rocha $\left(^{2}\right)$ \\ A. I. Reis Luz $\left({ }^{3}\right)$ \\ W. A. Rodrigues $\left({ }^{3}\right)$
}

\begin{abstract}
Resumo
Consta deste trabalho uma lista de espécies da familia Annonaceae, que possuem representantes no herbário do Instituto Nacional de Pesquisas da Amazônia, com os respectivos resultados do teste para indicação da presença ou não de alcalóides. Complementa o trabalho um levantamento da constituiçẫo químico-alcaloídica da familia em geral, dando-se ênfase especial aos gêneros mencionados para a América.
\end{abstract}

Em trabalhos anteriores, (Rocha et al. 1968 e 1979) apresentamos um "screening" de alcalóides em espécies botânicas da Amazônia registradas no herbário do INPA-Manaus. Neste trabalho, focalizaremos a familia Annonaceae e nos subseqüentes as demais familias onde foram observados resultados positivos, objetivando estabelecer o conhecimento químico-alcaloídico atual do taxon em geral e, em particular, de seus representantes na Amazônia, de modo a proporcionar bases para a racionalização de futuras pesquisas.

\section{AnNonaceae}

A família Annonaceae (Wa'ker, 1971) é constituída por, aproximadamente, 130 gêneros e 2.300 espécies. Filogeneticamente, é tida como angiosperma primitiva com um relacionamento próximo com famílias como Magnoliaceae e Myristicaceae. Fitogeograficamente, é quase inteiramente tropical, sendo os gêneros divididos em três centros - América (39 gêneros endêmicos), África, excluindo Madagascar (40 gêneros endêmicos) : Ásia (50 gêneros endêmicos). O gênero Xylopia ocorre nos três centros; os gêneros Uvaria, Polyantha e Artabotrys ocorrem na África e na Ásia; 0 gênero Anaxagorea aparece na Ásia e na América; e o gênero Annona existe na África e na América. Quimicamente, temos informações de somente 24 gêneros (ver tabelas) e destes apenas 7 estão representados no herbário do INPA-Manaus, que tem registrado 21 dos 39 gêneros mencionados para a América (ver Quadro I). Os tipos alcalóidicos de maior incidência são aporfina e oxoaporfina. No gênero Xylopia, é significante a presença dos tipos benziltetrahidroisoquinolina e protoberberínico (ver Tabelas).

QUADRO I - Gêneros que ocorrem na América:

\begin{tabular}{|c|c|}
\hline Anaxagorea (29)" & Guatteriella (1) \\
\hline Annona $(125)^{*}$ & Guatteriopsia $(4)^{\circ}$ \\
\hline Crematosperma $(17)^{*}$ & Heteropetalum $(2)^{*}$ \\
\hline Malmea $(14)^{*}$ & Dukeanthus $(1)^{*}$ \\
\hline Ephedranthus $(4)^{*}$ & Fusaea $(3)^{*}$ \\
\hline Pseudephedranthus (1) & Diclinanona (2) \\
\hline Pseudoxandra $(6)^{\circ}$ & Asimina $(8)$ \\
\hline Oxandra $(25)^{*}$ & Deeringothamnus (2) \\
\hline Ruizodendron (1) & Raimondia (4) \\
\hline Unonapsis $(33)^{\circ}$ & Rollinia $(65)^{*}$ \\
\hline Bocageopsis $(3)^{*}$ & Rolliniopsis (4) \\
\hline Onychopetalum $(4)^{*}$ & Cymbopetalum (13)* \\
\hline Desmopsis (16) & Cardiopetalum (1) \\
\hline Stananona (2) & Froesiodendron (2) \\
\hline Reedrollinsia (1) & Porcelia (5) \\
\hline Sapranthus (12) & Irigynaea (5) \\
\hline Iridimeria (1) & Bocagea (2) \\
\hline Tetrameranthus $(2)^{\circ}$ & Hornschuchia (3) \\
\hline Duguetia $(74)^{*}$ & Xylopia $(170)^{*}$ \\
\hline Guatteria $(250)^{*}$ & \\
\hline
\end{tabular}

(1) - Pesquisa financiada pelo Banco da Amazônia S.A.

(2) - Fundação Universidade do Amazonas, Manaus.

(3) - Instituto Nacional de Pesquisas da Amazônia, Manaus. 
As espécies de Annonaceae registradas no herbário do INPA-Manaus e os resultados dos testes para alcalóides constam do Quadro II.

\section{QUADRO ॥}

\section{ANNONACEAE}

N. herb.

FOLHA CAULE

Anaxagorea

-brachycarpa R.E. Fries

5784-brevipes Benth.

-pachypetala (Diels) R.E. Fries

- petiolata R.E. Fries

8966-phaeocarpa Mart.

Annona

5679-ambotay Aubl.

- crassifolia Mart.

- coriacea Mart.

8490 - echinata Dun

-excellens R. E. Fries

14111-foetida Mart.

- glabra L.

10860-hypoglauca Mart.

-hypoglauca Mart. var. longepedicellata Aristeguieta

3186-impressivenia Safford

- montana Macfad.

40908-muricata L.

3915-nitida Mart.

—paludosa Aubl.

-salzmannii DC.

6275-sericea Dun

- squamosa

Bocageopsis

21376-mattogrossensis R.E. Fries

5271-multiflora (Mart.) R.E. Fries

Cardiopetalum

-calophyllum Schlecht.

Crematosperma

-microcarpa R.E. Fries

Cymbopetalum

13949-brasiliense (Vell.) Benth.

-longipes Diels

Duckeanthus

7167-grandiflorus R.E. Fries

Duguetia

-amazonica R.E. Fries

-argentea R.E. Fries

14047-asterotricha (Diels) R.E. Fries

4340-calycena R. Ben.

- caudata R.E. Fries

6181-cuspidata R.E. Fries

-duckei R.E. Fries

-echinophora R.E. Fries

6529-eximia Diels

5751-flagellaris Huber

-latifolia R.E. Fries

$\begin{array}{ll}- & + \\ - & +\end{array}$

$+$
N. herb.

FOLHA

CAULE

47340 - marcgraviana Mart.

- oblanceolata R.E. Fries

-paraensis R.E. Fries

8647-pycnastera Sandw.

-quitarensis Benth

- spixiana Mart.

- spruceana R.E. Fries

14045-stelechantha (Diels) R.E. Fries +

8980 - surinamensis R.E. Fries $++\quad++$

4415-uniflora (Dun.) Mart.

Ephedranthus

8712-amazonicus R.E. Fries
Fusaea

-decurrens R.E. Fries

8642-longifolia (Aubl.) Safford.

Guatteria

-amazonica R.E. Fries

8992 -chrysopetala (Steud.) Miq.

-chrysopetala (Steud.) Miq. var. tenuipes R.E. Fries

4260 - citriodora Ducke

28961-dielsiana R.E. Fries

15480-discolor R.E. Fries

8476-duckeana R.E. Fries

-dura R.E. Fries

8289-foliosa Benth.

15803-guianensis (Aubl.) R.E. Fries

15195-insculpta R.E. Fries

9224-inundata Mart.

-kuhlmannii R.E. Fries

-longicuspis R.E. Fries

-maypurensis H.B.K.

13902-maypurensis H.B.K. var. attenuata H.B.K.

8933-megalophylla Diels

8956-megalophylla Diels var. deminuta R.E. Fries

5871-meliodora R.E. Fries

-micans R.E. Fries

5945-olivacea R.E. Fries

9247-ovalifolia R.E. Fries

-paraensis R.E. Fries

9008-phanerocampta Diels

- poeppigiana Mart.

-procera R.E. Fries

4343-pteropus Benth.

-riparia R.E. Fries

- scandens Ducke

4412-schomburgkiana Mart.

13328 - scythophylla Diels

-sessilis R.E. Fries

13156-spruceana R.E. Fries $\quad+++$

-trichoclonia Diels

Guatteriopsis

5707-blepharophylla (Mart.) R.E.Fries

9125-friesiana W. Rodrigues (n.sp.) +

- hispida R.E. Fries

-kuhimannii R.E. Fries

Rocha et al. 


\begin{tabular}{|c|c|c|c|c|c|}
\hline \multirow{2}{*}{$\begin{array}{l}\text { N. herb. } \\
\text { 9217-sessiliflora (Benth.) R.E. Fries }\end{array}$} & FOLHA & CAULE & N. herb. & FOLHA & CAULE \\
\hline & - & - & 3783-duckei R.E. Fries & + & + \\
\hline Heteropetalum & & & 4386-guatterioides (DC.) Fries & - & - \\
\hline -brasiliense Benth. & & & 14883-guatterioides (DC.) Fries $f$. & & \\
\hline Malmea & & & elongata Fries & - & - \\
\hline$-s p$ & & & -matthewsi (Benth.) R.E. Fries & & \\
\hline Onychopetalum & & & —spectabilis Diels & & \\
\hline 16128-amazonicum R.E. Fries & - & & 15718-stipitata Diels & ++ & \\
\hline Oxandra & & & 9449-williamsii R.E. Fries & + & + \\
\hline -asbecki (Pulle) R.E. Fries & & & Xylopia & & \\
\hline -euneura Diels. & & & 4128-amazonica R.E. Fries & - & - \\
\hline -krukoffii R.E. Fries & & & 8575-aromatica (Lam.) Mart. & + & - \\
\hline -polyantha R.E. Fries & & & 8595-barbata Mart. & + & + \\
\hline -riedeliana R.E. Fries & & & 5800 - benthami R.E. Fries & - & - \\
\hline Pseudoxandra & & & 15835-brasiliensis Spreng. & - & \\
\hline 9619-coriacea R.E. Fries & - & & -calophylla R.E. Fries & & \\
\hline 8297-guianensis R.E. Fries & ++ & - & 17293-crinita R.E. Fries & - & \\
\hline 9022-leiophylla (Diels) R.E. Fries & - & - & -discreta (L.f.) Sprague et & & \\
\hline 13507-polyphleba (Diels) R.E. Fries & +++ & & Hutchins & & \\
\hline Rollinia & & & —emarginata Mart. & & \\
\hline 8917-exsucca (Dun.) DC. & - & - & 10191-emarginata Mart. var. duckei & & \\
\hline 8139 -insignis R.E. Fries var. pal- & & & R.E. Fries & - & - \\
\hline lida R.E. Fries & - & - & -frutescens Aubl. & & \\
\hline -mucosa (Jacq.) Baill. & & & -frutescens Aubl. var. ferru- & & \\
\hline 14227-prancei Aristeguieta & - & & ginea R.E. Fries & & \\
\hline Tetrameranthus & & & -grandiflora St. Hil. & & \\
\hline 6232-duckei R.E. Fries & - & - & - manauensis Aristeguieta & & \\
\hline Unonopsis & & & 一nitida Dun. & & \\
\hline -buchtienii R.E. Fries & & & -prancei Aristeguieta & & \\
\hline
\end{tabular}

\section{TABELAS}<smiles>CCCc1cccc2c1ccc1ccccc12</smiles>

Tipo fenantrênico
$\mathrm{NR}_{2}$

Gênero
Enantia
Monodora
Annona
Enantia
Monodora
Meiocarpidium
Uvariopsis
$\quad "$

$n$<smiles>COc1cc2c3c(cc4ccccc4c3c1OC)C(=O)[IH]2</smiles>

Gênero
Schefferomitra

Substância

cepharanona

B=aristolactama B II
Referência

$72,82,87$

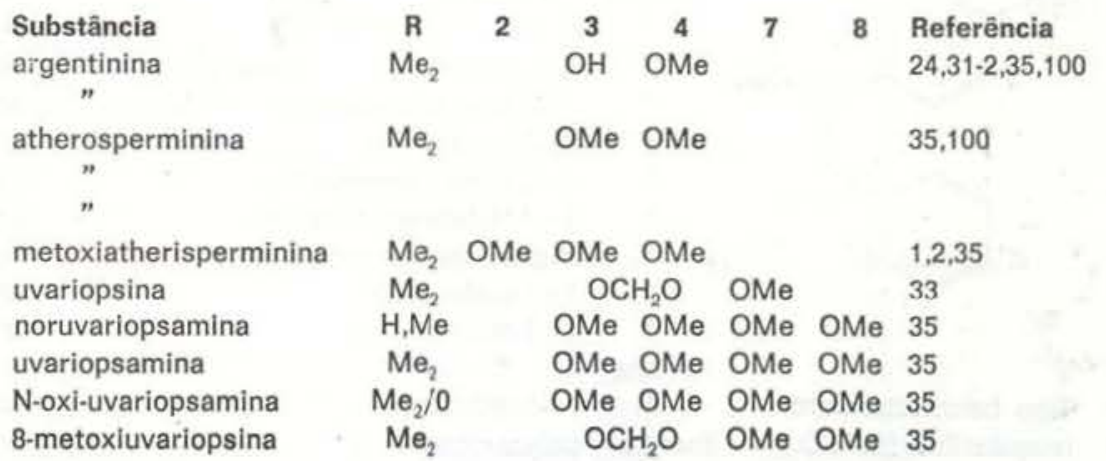



$R_{1} \quad R_{2}$
(a) - $\mathrm{OMe} \mathrm{OMe}$
(b).$--\mathrm{OCH}_{2} \mathrm{O}$.<smiles>[R]c1cc2ccnc3c2c(c1[R2])-c1ccccc1C3=O</smiles>

Tipo oxoaporfina

$\begin{array}{cccl}3 & \mathbf{9} & \mathbf{1 0} & \begin{array}{l}\text { Referência } \\ 100-1\end{array} \\ & & 35,66,71,80,100 \\ \mathrm{OMe} & & 107 \\ & & 35 \\ \mathrm{OH} \mathrm{OMe} & 2,7,26-7,35,63,102 \\ & \mathrm{OMe} \mathrm{OMe} & 26\end{array}$

Referência $92-4,97,102$

\section{Gênero \\ Annona \\ Asimina \\ Cananga \\ Mitrela \\ Melodorum \\ Pseudovaria \\ Polyalthia \\ Schefferomitra \\ Uvariopsis \\ Enantia \\ Xylopia \\ Fusaea \\ Guatteria$$
\text { " }
$$ \\ Enantia \\ Polyalthia \\ Uvariopsis \\ Enantia \\ Polyalthia \\ Xylopia \\ Guatteria<smiles>COc1ccc2c(c1)CCNC2Cc1ccccc1</smiles>

Substância

lysicamina $=$ oxonuciferina

O-metilmoschatolina $=$ liridi-

na =homoschatolina

"

subsessilina

O-metilatherolina $=$ liriodendron

base $=$ oxoglaucina

oxopurpureina

\section{Substância}

liriodenina $=$ spermatheridina $=$

oxoushinsunina $=$ michelina $B$

$$
\begin{aligned}
& " \\
& n \\
& n \\
& n \\
& n \\
& n \\
& n \\
& n \\
& n \\
& n
\end{aligned}
$$

$n$

n

$n$

$n$

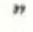

$n$

$n$

atherospermidina $=$ psilopina

oxostephanina

8,9-dimetoxiliriodenina

lanuginosina $=0$ xoxylopsina

n

oxoputerina

$\begin{array}{cl}\begin{array}{c}\text { Gênero } \\ \text { Xylopia }\end{array} & \begin{array}{l}\text { Substância } \\ \text { coclaurina } \\ n\end{array} \\ n & (+) \text {-isococlaurina } \\ n & (-)-N \text {-nor-O-metilcoclaurina } \\ n & (-) \text {-armepavina } \\ n & (-)-O-\text {-metilarmepavina } \\ n & (-) \text {-N-desmetilcolletina } \\ n & \text { N-oxi-O-metilarmepavina } \\ n & ( \pm) \text {-laudanidina } \\ n & (+) \text {-reticulina } \\ \text { Annona } & n \\ n & (-) \text {-reticulina } \\ \text { Enantia } & \text { polycarpina }\end{array}$

\section{$\mathrm{OMe}$ \\ $\mathrm{OMe} \mathrm{OMe}$ \\ $\mathrm{OMe}$}

Tipo benziltetrahidroisoquinolina (B.T.I.Q.)

\begin{tabular}{|c|c|c|c|c|c|}
\hline R & 7 & $2^{\prime}$ & 3' & $4^{\prime}$ & Referência \\
\hline H & $\mathrm{OH}$ & & & $\mathrm{OH}$ & $2,4,5,6$ \\
\hline $\mathrm{H}$ & $\mathrm{OH}$ & & & $\mathrm{OH}$ & $1-4,7$ \\
\hline $\mathrm{H}$ & $\mathrm{OMe}$ & & & $\mathrm{OMe}$ & 9 \\
\hline $\mathrm{Me}$ & $\mathrm{OH}$ & & & $\mathrm{OH}$ & 9 \\
\hline $\mathrm{Me}$ & $\mathrm{OMe}$ & & & $\mathrm{OH}$ & 9 \\
\hline $\mathrm{Me}$ & OMe & & & $\mathrm{OMe}$ & 9 \\
\hline $\mathrm{Me}$ & $\mathrm{OH}$ & & & $\mathrm{OMe}$ & 9 \\
\hline $\mathrm{Me}, \mathrm{O}$ & $\mathrm{OMe}$ & & & $\mathrm{OMe}$ & 9 \\
\hline $\mathrm{Me}$ & $\mathrm{OMe}$ & & $\mathrm{OH}$ & $\mathrm{OMe}$ & 9 \\
\hline $\mathrm{Me}$ & $\mathrm{OH}$ & & $\mathrm{OH}$ & $\mathrm{OMe}$ & $9-12$ \\
\hline $\mathrm{Me}$ & $\mathrm{OH}$ & & $\mathrm{OH}$ & $\mathrm{OMe}$ & 58 \\
\hline $\mathrm{CHO}$ & $\mathrm{OMe}$ & $\mathrm{OH}$ & OMe & $\mathrm{OMe}$ & 66,98 \\
\hline
\end{tabular}

\section{$\mathrm{OMe}$}

105 
<smiles></smiles><smiles>COc1cc2c(cc1OC)C(Cc1ccccc1)N(C)CC2</smiles>

Tipo bis B.T.I.Q.

\begin{tabular}{|c|c|c|c|c|c|c|c|c|c|}
\hline Gênero & Substância & 6 & 7 & 12 & $7^{\prime}$ & 12 & $\Delta$ & Ponte & Referência \\
\hline Phaeanthus & phaeantharina & $\mathrm{OMe}$ & & & OMe & $\mathrm{OMe}$ & $\begin{array}{l}1,3 \\
1^{\prime}, 3\end{array}$ & $\begin{array}{l}7-0-8^{\prime} / \\
12-0-11^{\prime}\end{array}$ & 1,20 \\
\hline$n$ & $\begin{array}{l}(-) \text {-tetrandrina }= \\
\text { phaeanthina }\end{array}$ & OMe & & & OMe & $\mathrm{OMe}$ & & $n$ & $1,3,8,17,20$ \\
\hline Crematosperma & phlebicina & $\mathrm{OH}$ & & $\mathrm{OH}$ & OMe & $\mathrm{OMe}$ & & $\begin{array}{l}7-0-8^{\prime} / \\
11-11^{\prime}\end{array}$ & 44,87 \\
\hline Isolona & $\begin{array}{l}\text { bebeerina }=\text { cucina } \\
=\text { chondodendrina }\end{array}$ & $\mathrm{OMe}$ & & & $\mathrm{OH}$ & $\mathrm{OH}$ & & $\begin{array}{l}7-0-11^{\prime} / \\
12-0-8^{\prime}\end{array}$ & 21,79 \\
\hline Uvaria & chondrofolina & OMe & & & OMe & $\mathrm{OH}$ & & $"$ & $1,2,21,68$ \\
\hline $\begin{array}{c}\text { Guatteria } \\
\text { " }\end{array}$ & $\begin{array}{l}(--)-0, \text { O-dimetilcurina } \\
(--)-12 \text {-O-metilcurina }\end{array}$ & $\begin{array}{l}\mathrm{OMe} \\
\mathrm{OMe}\end{array}$ & & & $\begin{array}{l}\mathrm{OMe} \\
\mathrm{OH}\end{array}$ & $\begin{array}{l}\mathrm{OMe} \\
\mathrm{OMe}\end{array}$ & & $"$ & $\begin{array}{l}81 \\
81\end{array}$ \\
\hline$"$ & (- - ) -isochondodendrina & $\mathrm{OMe}$ & $\mathrm{OH}$ & & $\mathrm{OH}$ & & & $\begin{array}{l}8-0-12^{\prime} / \\
12-0-8^{\prime}\end{array}$ & 81 \\
\hline Isolona & $\begin{array}{l}\text { ( )-isochondodendrina }= \\
\text { ( )-isobebeerina }\end{array}$ & $\mathrm{OMe}$ & $\mathrm{OH}$ & & $\mathrm{OH}$ & & & $"$ & $3,7,48,54,67,79$ \\
\hline
\end{tabular}<smiles>COc1cc2c3c(c1)C1CC(C=CC3=O)CCC(CCN1)C2</smiles>

Tipo proaporfina
\begin{tabular}{ll}
$R_{1}$ & $R_{2}$ \\
\hline
\end{tabular}
(a) $-\mathrm{H} \quad \mathrm{H}$
(b) $-\mathrm{OH} \quad \mathrm{OMe}$
(c) $-\mathrm{OMe} \mathrm{OH}$
(d) $-\mathrm{OMe} \mathrm{OMe}$
(e) $-\cdot \mathrm{OCH}_{2} \mathrm{O}$ -

\begin{tabular}{cllll} 
Gênero & Substância & $\mathbf{R}$ & \multicolumn{1}{c}{} & Referência \\
Annona & (+)-glaziovina & $\mathrm{Me}$ & $\mathrm{OH}$ & $8,13,17,26$ \\
$n$ & (+)-stepharina & $\mathrm{H}$ & $\mathrm{OMe}$ & $2,3,8,13,26,36,37$ \\
Isolona & pronuciferina & $\mathrm{Me}$ & $\mathrm{OMe}$ & 49,79
\end{tabular}

(a)

Gênero
Enantia

(b)

$\begin{array}{ll}\begin{array}{l}\text { Gênero } \\ \text { Isolona } \\ \text { " }\end{array} & \begin{array}{l}\text { Substância } \\ \text { caaverina } \\ \text { isopilina }\end{array} \\ \text { Monodora } & \begin{array}{l}(+) \text {-sparsiflorina } \\ \text { Isolona }\end{array} \\ \text { zenkerina } \\ \text { Popowia } & \text { wilsonirina=aducaina }\end{array}$

Substância magnoflorina $=$ thalictrina $=$ esholina $=$ escholina<smiles>[R2]c1cc2c3c(c1[R2])-c1ccccc1CC3[Y]C(=O)C2</smiles>

Tipo aporfina

Referência

101,104

$\begin{array}{cccccl}3 & \text { R } & \mathbf{9} & \mathbf{1 0} & \mathbf{1 1} & \text { Referência } \\ & \mathrm{H} & & & & 35,79 \\ \mathrm{OMe} & \mathrm{H} & & & & 79 \\ & \mathrm{H} & & \mathrm{OH} & & 79 \\ & \mathrm{H} & & \mathrm{OMe} & & 79 \\ & \mathrm{H} & \mathrm{OMe} & \mathrm{OMe} & & 7,35\end{array}$




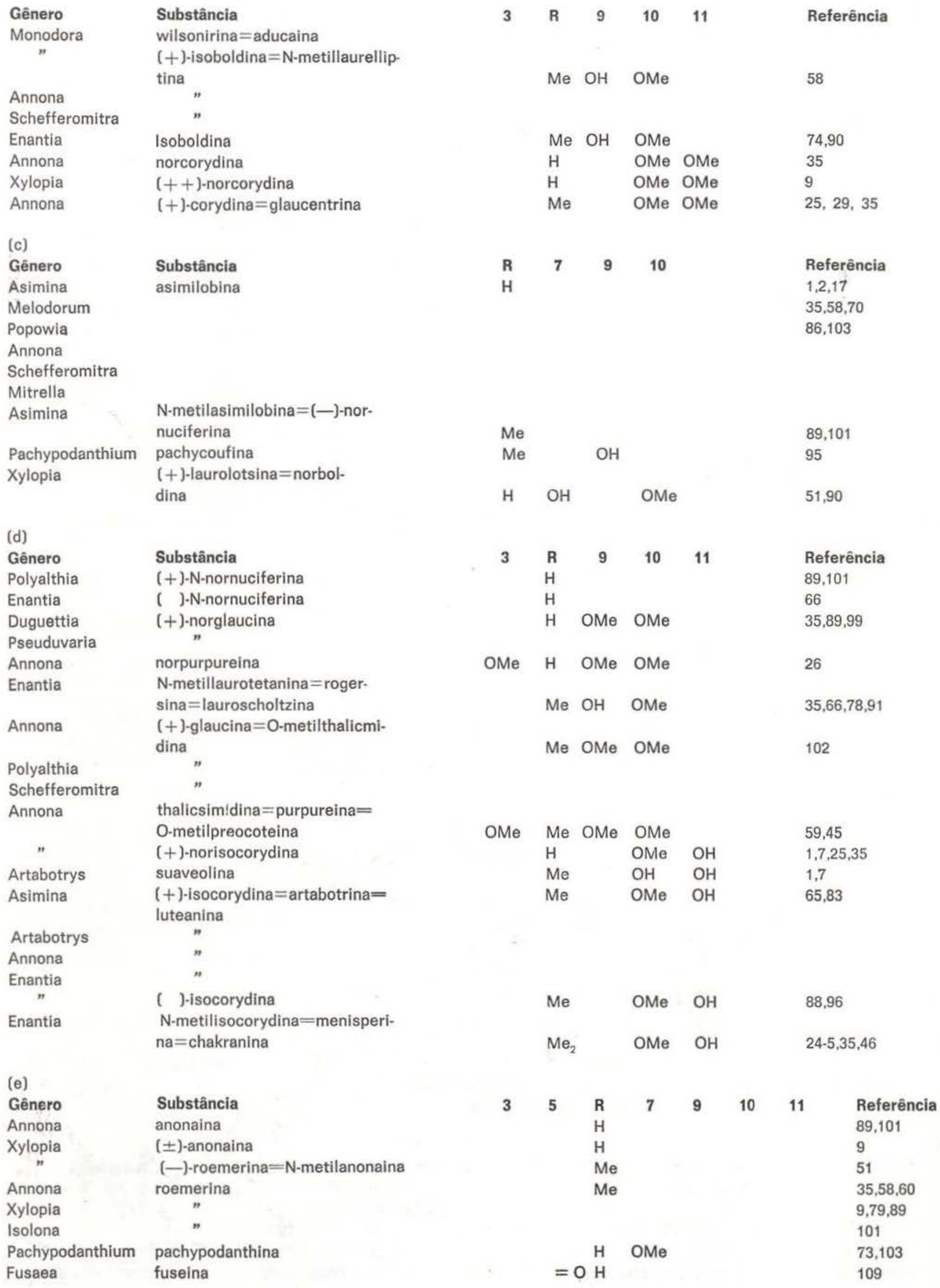




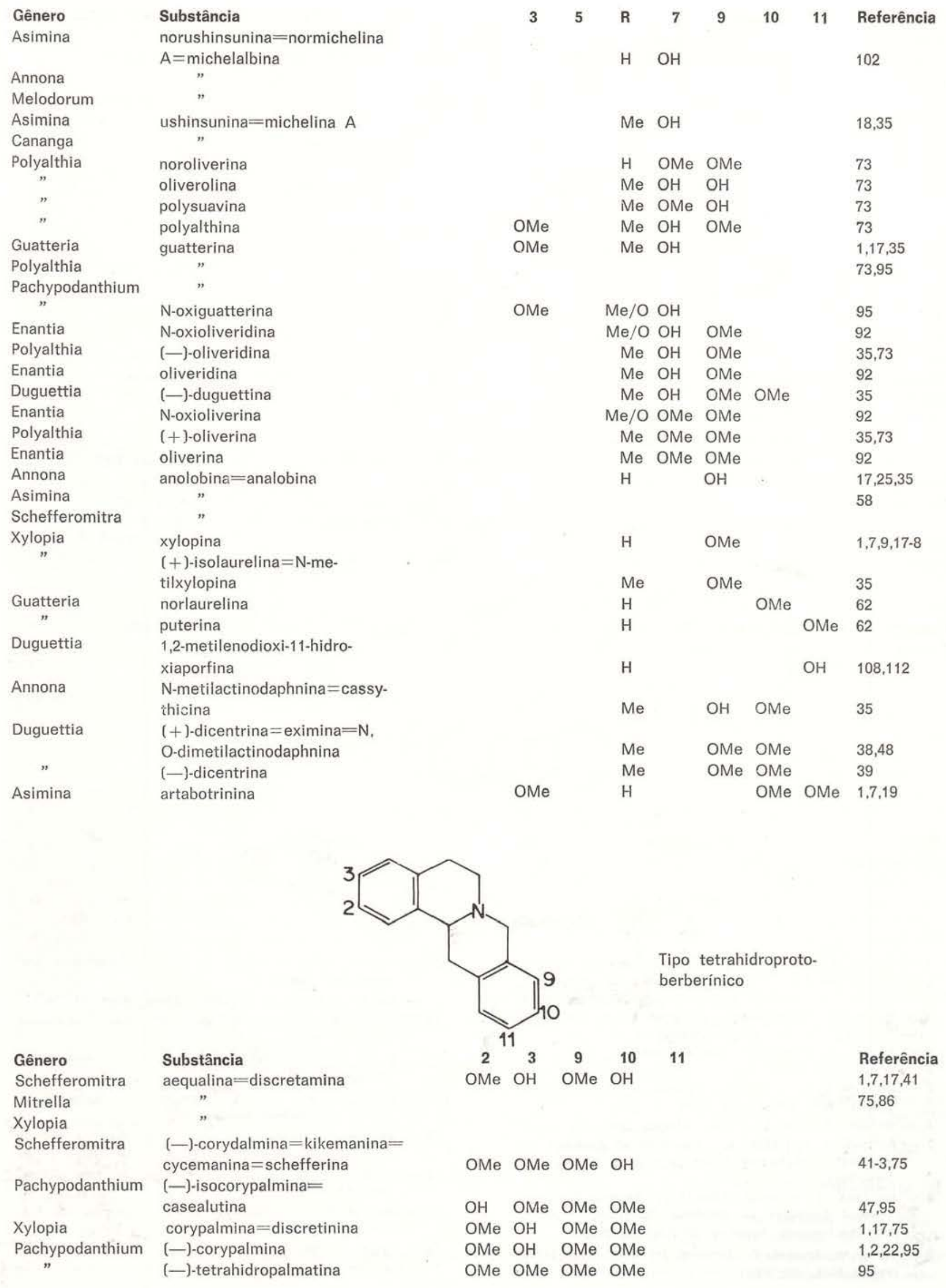




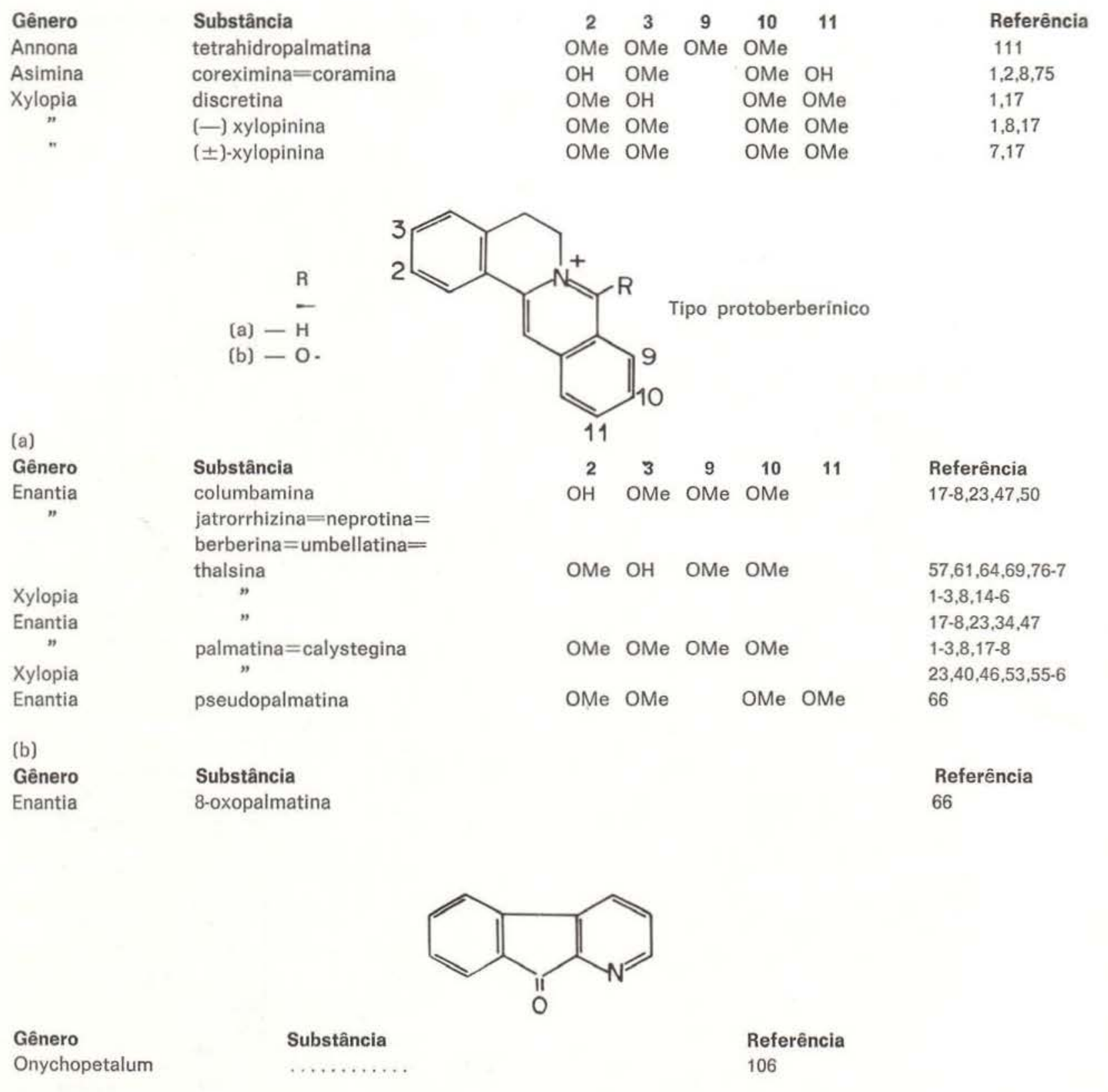

\section{REFERENCIAS DAS TABELAS}

1 - Darnley Gibbs, R, (1974) Chemotaxonomy of Flowering Plants, Mc Pill - Queen's University Press, Montreal.

2 - Shomma, M. (1972) The isoquinoline Alkoloids, Chemistry and Phormacology, Academic Press, New York.

3 - Thornber, C. W. (1970) Phytochem, 9, 157.

4 - Manske, R. H. F. (1968) The Alkaloids vol. X, Acodemic Press, New York.

5 - Bhakuni, D. S., Gonzales \& Sammes, P. G. e Silva, M. (1974) Rev. Latinoamer. Quim, 5, 158.

6 - William, J. J. e Li (1970) Lloydia Supl. 33, I.

7 - Roffouf, R. F. [1970) A. Handbook of Alkaloids and Alkaloid - containnig Plants John Wiley \& Sons, Inc., New York.

8 - Holubeck, J. e Strouf (1964-1972) Spectral Data and Physical Constants of Alkaloids, Publishing House of the Czechoslovak Academy of Sciences, Prague.

9 - Nieto, M., Sevenet, T., Leboenf, M. e Cave, A. (1976) Planta Med., 30, 48.

\footnotetext{
10 - Hansen, E. B. e Nilsen, B. (1965) Tetrahedron Letters, 1271.

11 - Lu, S-T, e Horng, C. J. (1978) Chem. Abst. 88, 34561.

12 - Urzua, A. e Cassels, B. K. (1977) Rev. Latinoamer. Quim. 8. 133

13 - Stuart, K. L. O Cava, M. P. (1968) Chom, Rov. 68,321 .

14 - Brown, D. W., Dyke, S. F., Hardy, G. e Sainsbury, M (1969) Tetrahedron Letters 1515.

15 - Monkovic, I. e Spencer, I. D. (1965)..Can. J. Cher: 43, 2017.

16 - Monkovic, I, e Spencer, I, D. (1965) J. Ame. Chem, Soc. $87,1137$.

17 - Manske, R. H. F, (1970) The Alkaloids vol, IX, Academic Press, New York.

18 - Schiff Jr., P. L, e Doskotch, R. W. (1970) Lloydia $33,403$.
} 
19 - Boit, H. G. (1961) Ërgebnisse der Alkaloid Chemie bis 1960;, Akademic-Verlag, Berlin.

20 - Manske, R. H. F. (1960) The Alkoloids, vol, VII, Aco. demic Press, New York.

21 - Manske, R. H. F. (1954) The Aftxaloids, vol. IV. Acodemic Press, New York.

22 - Chan, K, C., Evons, M. T. A. Hassel, C. H. e Sangster, A. M. W. (1967) J. Chem. Soc. (C) 2479.

23 - Bhakuni, D. S., Shoeb, A. e F opli, S. P. (1968) Indian J. Cherm. 6, 1:3.

24 - Munavalli, S. e Viel, C. (1969) Ann. Phamn. (Fran. ce) $27,449,519,601$ e 610 .

25 - Shamma, M. e Slusarchyk, N. A. (1964) Chem. Rev. $64,1254$.

26 - Sonnet, P. E. e Jacobson, M. (1971) J. Pharm. Sci. 60,1254 .

27 - Cava, M. P.. Roo, K. V., Douglas, B. e Weisbach, J. A. (1968) J. nrg. Chem. 33, 2443

28 - Kupchan, S. M., Suffness, M. I. e Gordon, E. M (1970) J. Org. Chem. 35, 1682,

29 - Shammo, M. e Slusarchyk, W. A. (1967) Chem. Abst. 67, 54294.

30- Bick, 1. R. C. e Douglas, G. K. (1964) Tetrohedron Letters 1629

31 - Priestap, H. A., Ruvedo, E. A. e Albonico S. M. (1967) Chem. Comm, 754.

32 - Priestap, H. A., Ruvedo, E. A., Albonico, S. M. e Denlofen, V. (1972) An. Assor, Quim. Arg. 60, 309.

33 - Bouquet, A., Cove, A., Cace, A. e Paris, R. R, (1970) C. R. Acad. Sci. (Paris), ser. C 271, 1100.

$34-\mathrm{Kim}, \mathrm{H}$. K. Farnsworth, N. R., Blomste, R. N. e Fong, H. H. S. (1969) J. Pharm. Sci, 58, 372.

35 - Guinodeau, H., Leboeuf, M, e Cave, A. (1975) Lloydia 38,275 .

36 - Tomito, M., Okomoto, Y.. Nagai, Y., Tanaka, 5, e Hayata, T. (1971) Chem. Abstr. 74, 1113d.

37 - Yang, T.H. Lin, S.-C. (1974) Chem. Abstr., 81, $60844 \mathrm{k}$.

38 - Patnaik, P. C. e Gopinath, K. W. (1975) Indian J. Chem 13, 197

39 - Casagradne, C. e Ferrari, G. (1970) Chem. Abstr., 73, $38472 a$.

40 - Panov, P. P., Panova, L. N. e Mollov, N. M. (1972) Chem. Abstr. 77, 72554w.

41 - Gellert, E. e Rudizats, R, (1972) Aust, J. Chem. 25, 2477.

42 - Kametani, T., lhara, M. e Aonda, T. (1969) J. Chem. Soc. D 1301.

43 - Sekolniko, L. 1. (1971) Chem, Abstr, 74, 1075t.

44 - Cava, M. P., Wakisaka, K., Naguchi, I., Edie, D. L. e Da Rocha, A. I. (1974) J. Org. Chem. 39, 3588.

45 - Shamma, M. e Podczazy, St. M. A. (1971) Tetrahedron 27,727 .

46 - Tani, C., Hogakura, N. e Hattori, S. (1075) Yakugaku Zasshi 95, 1103.

47 - Slavik, J. e Slavikova, L. (1976) Chem. Abstr. 84, 2274.

48 - Dwnma-Badu, D., Ayine, J. S. K., Mingle, C. A., Toc. kie, A. H., Slatkin, D. J., Knapp, J. E, e Schiff, P. H. Jr. (1975) Phytochemistry 14, 2520.

49 - Hemangway, S. R. e Phillipson, J. D. (1975) J. Phorm. Pharmecol. 27 suppl. $84 \mathrm{p}$

50 - Wu, W.-N., Beal, J. L., Clark, G. W. e Mitscher, L. A. (1976) Lloydia 39,65 .

51 - Lu, J.-T., Su, T.-L. e Wang, E.-C. (1976) Chem Abstr, 84, 132659r.

52 - Nieto, M., Leboeuf, M. e Cave, A. (1975) Phytzchemistry 14, 2508.

53 - Tani, C., Tagahara, K. e Aratani, S, (1976) Yakugaku Zasshi $96,527$.
54 - Gupta, D. B., Banergee, S. K. e Handa, K. L. (1976) Phytochemistry 15, 576.

55 - Karimov, A., Telezhenetskaya, M. V. e Hatfullin, K. (1976) Chem. Abstr. 84, 14662.

56 - Chen., I.-S. (1976) Chem. Abstr. 84, 28054.

57 - Baytop, T. e Berghmons, M. (1976) Chem. Abstr. 84,40710 .

58 - Yan, T. . H. e Chen, C. - M, (1976) Chem, Abstr. 84,102339 .

59 - Maekh, S., Gorovoi, P. G. e Yunusov, S. Yu. (1976) Chem. Abstr, 85, 174274.

60 - Zelenski, S. G. (1977) J. Pharm. Sci. 66, 1627.

$\$ 61$ - Wu, W.-N., Beal, J. L, e Doskotch, R. W. (1977) Lolydia 40,508 .

62 - Hsu, C. C., Dobberstein, R. H., Cordell, G. A. e Farnsworth, N. R. (1977) Lloydia 40, 505

63 - Shafiee, A., Lalezari, I, e Mahiour, M. (1977) J. Pharm. Sci., 66, 593.

64 - Kostalova, D., Brazdovicovo, B. e Tomko, J. (1977) Chem. Abstr. 86, 185942.

65 - Chen, C.-H., e Wu, J. (1978) Chem. Abstr. 88, 34563 .

66 - Jossang, A., Leboeuf, M, e Cave, A. (1977) Planta Med. 32, 249 .

67 - Dahmen, K., Pochaly, P. e Zymalkowski, F. (1977) Arch. Pharm. 310, 95 .

68 - Panichpol, K.. Waigh, R. D e Waterman, P. G. (1977) Phytochemistry 16,621.

69 - Brozodicova, B., Kostalova, D e Zubakova, A. (1977) Chem. Abstr, 87, 81269.

70 - Ziyaev, R., Irgaslev, T.. Israilov, I. A., Abdullaev, N. D., Yunusov, M. S. e Yunusov, S. Yu. (1977) Chem. Abstr. 87, 114612

71 - Cava, M. P., Buck, K. T., Noguchi, 1., Sirinivan, M., Roo, M, G. e Da Rocha, A. I. (1975) Tetrahedron 31, 1667 .

72 - Dyke, S. F. e Gellert, E. (1978) Phytochemistry 17, 599.

73 - Cave, A., Guimanciean, H.. Leboeuf, M. Ramahatra, A, e Razafindrazaka, J. (1978) Planta Medica, 33, 243.

74 - Israilov, I. A, Irgaslev, T. Yunusov, M. S. e Yunusov, S. Yu (1977) Khim. Prir. Socdin. 834.

75 - Brochmann.Hanssen, E. e Chiang, H.-C. (1977) J, Org. Chem. 42, 3588 .

76 - Adesina, S. K., Harborne, J. B. e Phillipson, I. D. (1978) Planto Medica 33, 217.

77 - WU, W.-N., Beal, J. L., Fairchild, E', H. e Doskotch, R. W. (1798) J. Org. Chem, 43, 580 .

78 - Wu, J., Beal, J. L., Wu, W. - N. e Doskatch, R. W. (1977) Lloydia 40, 593.

79 - Hocquemiller, R., Cobalion, P., Bouquet, A. e Cave, A. (1977) C. R. Hebd. Seances Acad, Jci. Ser C. 285, 447.

80 - Dwumie- Bodu, D., Ayim, J. S, K., Tackie, A. N.. Knapp, J. E., Slatkim, J. D. e Schiff, P. L Jr, (1975) Phytochemistry 14, 2524.

81 - Galeffi, C., Marini-Bettolo, G. B. e Vecchi, D. (1975) Gaxz, Chim. Ital, 105, 1207.

82 - Akosu, M., Ifokawo, H. e Fujito, M. (1974) Tetrahedron letters 3609

83 - Franca, N. C., Giesbrecht, A. N., Gottlieb, O. R., Magalhăes, A. F., Mogalhăes, E. G, e Maio, J. G. S. (1975) Phytochemistry 14, 1671 .

84 - Watanabe, Y.. Matsui, M.. libuchi, M. e Hiroe, S. (1975) Phytochemistry 14, 2522.

85 - leboeuf, M. e Cové, A. (1972) Phytochemistry 11, 2833.

86 - Saxton, J. E., ed. (1974) The AlKaloids vol. 4 (Specialist Prediodical Reports), The Chemical Society, London.

87 - Grudon, M. F., ed, (1976) The Alkaloids vol. 6 (Special-; ist Prediodical Reports), The Chemical Society, London. 


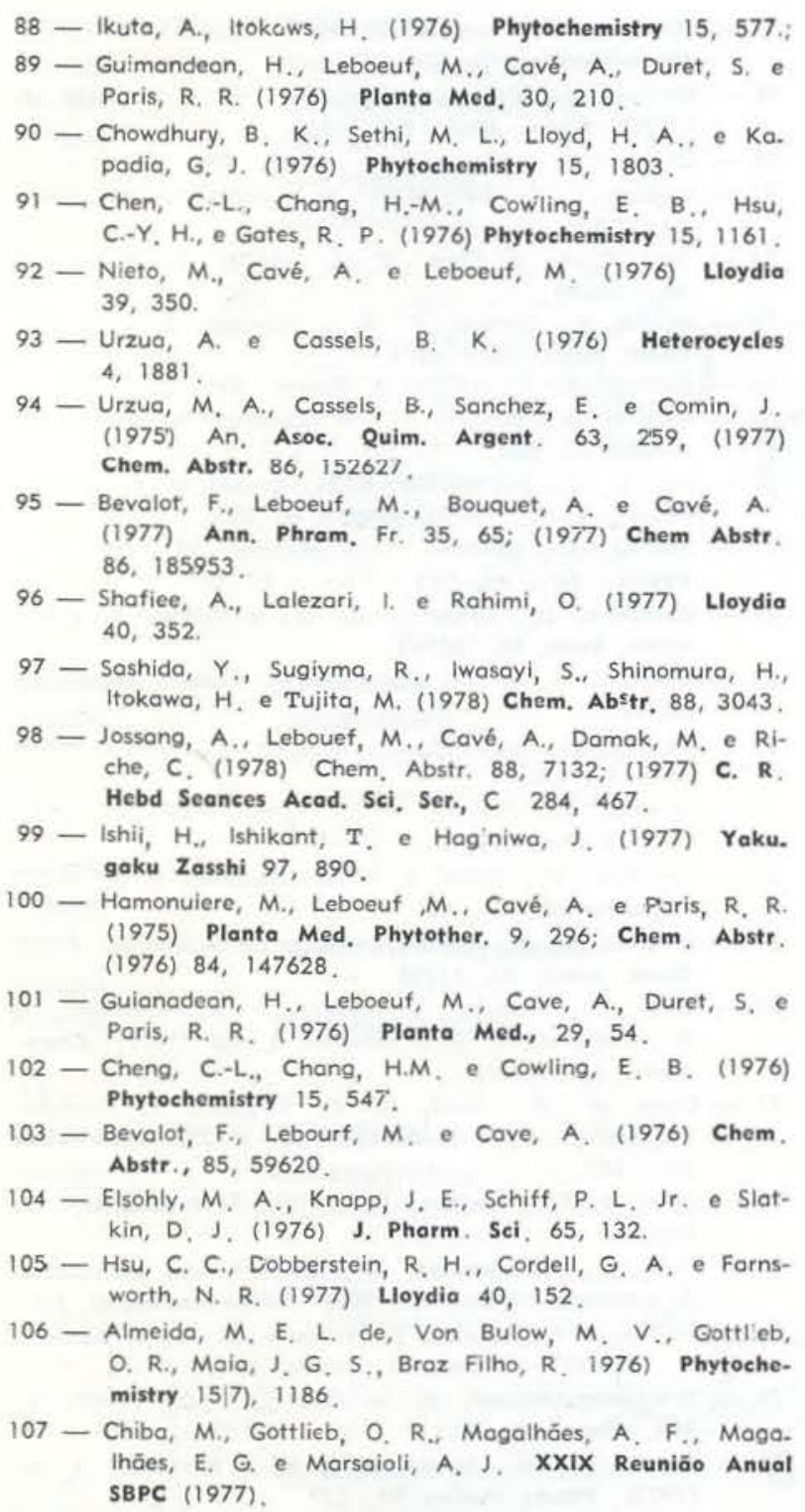

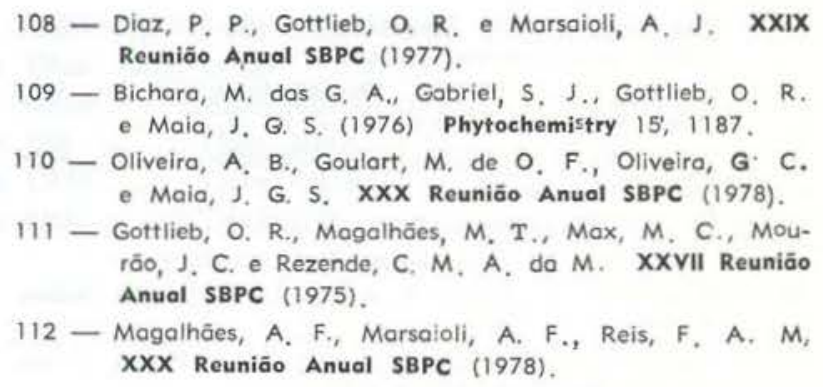

\section{SUMMARY}

A list of Annonaceae species from the Instituto Nacional de Pesquisas da Amazônia herbarium is presented with the respective results of the indication test of the presence or not of alkaloids. A avaliation of the chemical-alkaloidical constituints of the thorough family. standing out specially the american genera, is included too.

\section{REFERENCIAS BIBLIOGRAFICAS}

ROCHA, A.I. da; SILVA, M.L. da; MOURĀO, A.P. \& CAVA, M.P.

1968 - A Presença de Alcalóides em Espécies Botânicas da Amazônia. Boletim, n.`12. Série Química, INPA, Manaus. (12).

ROCHA, A.I. da \& REIS LUZ, A.I.

1979 - A Presença de Alcalóides em Espécies Botânicas da Amazônia. II. In: XXXI Reunião da SBPC, Fortaleza (CE).

WALKER, J.M.

1971 - Pollen Morphology, Phytogeography and Phylogeny of the Annonaceae. Contribuitions Gray Herbarium (202).

(Aceito para publicação em $26 / 11,80$ ) 